\title{
Mechanically supporting uterosacral ligaments for the relief of provoked vulvodynia: A randomised pilot trial
}

\author{
Matan Schonfeld ${ }^{1}$, Peter Petros ${ }^{2}$, and Jacob Bornstein ${ }^{3}$ \\ ${ }^{1}$ Bar-Ilan University \\ ${ }^{2}$ University of Western Australia \\ ${ }^{3}$ Galilee Medical Center
}

September 16, 2020

\begin{abstract}
Objective: Provoked vulvodynia (PV) is the main cause of vulvar pain and dyspareunia. Although its cause is unknown, it is associated with musculoskeletal dysfunction. The inability of lax uterosacral ligaments (USLs) to support the adjoining T11/L2 and S2-4 nerve plexuses is considered to cause PV. We aimed to determine whether mechanically supporting the USLs would reduce PV. Design: Single-blind, randomized, sham, control, prospective, pilot trial Setting: The Department of Obstetrics and Gynecology, Galilee Medical Center, Israel Population or Sample: Seventeen women Methods: PV patients were randomly assigned to two groups. Subjects in each group were exposed to sham manipulation (inserting a wide swab in the vagina without applying pressure) and trial manipulation (supporting the posterior fornix with a wide swab sufficiently broad to mechanically support the USLs). The manipulation order was alternated. Main Outcome Measures: Using a pain intensity scale, the PV-associated pain level experienced by participants was recorded during each manipulation and the results were compared with baseline levels. Results: The pain level significantly reduced with USL support compared with the baseline value and sham manipulation pain level $(\mathrm{P}=.003)$. Pain during sham manipulation was not significantly different from that recorded at baseline. The average reduction in pain with USL support was $18.4 \% \pm 2.2 \%$. The manipulation order did not impact changes in pain level during trial manipulation $(\mathrm{P}=.512)$. Conclusions: $\mathrm{PV}$ is related to pain originating in the USL and musculoskeletal dysfunction.
\end{abstract}

\section{Introduction}

Provoked vulvodynia (PV) is an idiopathic condition of chronic vestibular allodynia that leads to superficial pain, entry dyspareunia, and sexual dysfunction. ${ }^{1}$ It significantly reduces patients' quality of life and has a lifetime prevalence of approximately $9.9 \%{ }^{2}$ Although vulvodynia has no clear identifiable cause, ${ }^{1}$ a recent consensus acknowledged that vulvodynia may be associated with several factors. ${ }^{1}$

Although neuroproliferation is a possible cause of $\mathrm{PV},{ }^{3}$ musculoskeletal pelvic floor dysfunction has also been associated with PV, and physical therapy aimed at pelvic floor rehabilitation has been useful. ${ }^{4}$ However, the exact pathogenesis of pelvic floor instability and its association with the development of vulvodynia have not yet been elucidated. We propose that laxity of the uterosacral ligaments (USLs) in pelvic floor disorder in vulvodynia occurring without overt pelvic organ prolapse could trigger the development of PV. The USLs normally support the T10-L1-2 Frankenhauser sympathetic plexus or sacral S2-4 parasympathetic plexus (Figure S1). ${ }^{5}$ Loss of USL support of the Frankenhauser plexus has been associated with chronic pelvic pain of unknown origin (CPPU). ${ }^{5,6}$ Our hypothesis that PV, like CPPU, may be referred pain from unsupported nerve plexuses originated from the observation of three women with CPPU and vulvodynia who were cured by a posterior sling procedure, ${ }^{7,8}$ and also by studying 10 women with PV who were administered an injection of $2 \mathrm{~mL}$ local anesthetic in the USLs at their insertion points to the cervix, which are the estimated anatomical 
sites of the nerve plexuses. ${ }^{9} \mathrm{PV}$ was completely relieved for 30 minutes. For eight of these women, the pain temporarily disappeared completely on both sides; for two women, the pain disappeared on one side only. ${ }^{9}$

These findings were intriguing because the sensory nerve fibers to the vestibule branch have been reported to exit from the pudendal nerve, mainly through its perineal branch. The route of this nerve from its origin in S2-4 through the pelvis to the vestibule is not associated with or supported by the USL. ${ }^{7}$ Nevertheless, nerve fibers originating in the Frankenhauser plexus do terminate very close to the vestibule, clitoris, Bartholin's gland, and distal vagina ${ }^{7}$; therefore, they may be involved in the allodynia and hypersensitivity involved with PV. This pilot study aimed to provide temporary mechanical support to the USLs to further test our hypothesis that the cause of PV is the inability of the lax USLs to support the Frankenhauser and sacral plexuses stimulated by gravity to cause pain.

\section{Methods}

This study was a prospective, single-blind, randomized, controlled, pilot trial using a within-subject crossover design. The study was approved by the institutional review board of the Galilee Medical Center of the Israeli Health Ministry, on July 23, 2017; authorization number: 0043-17-NHR. ClinicalTrials.gov Identifier: NCT03197337. Written informed consent was obtained from all subjects.

Participants were women 18 to 35 years of age who met Friedrich's first two criteria ${ }^{10}$ for vulvar vestibular syndrome (referred to here as PV): severe pain in the vulvar vestibule on touch or attempted vaginal entry and tenderness to pressure (i.e., with a Q-tip applicator) localized within the vulvar vestibule. We included only women who were diagnosed with either moderate or severe PV (able to have sexual intercourse but with immense pain or unable to have sexual intercourse at all). ${ }^{11}$ Patients were excluded if they had vulvar pain caused by a specific disorder (such as that defined by the 2015 of the consensus terminology), ${ }^{1}$ had been diagnosed with generalized vulvodynia, had been previously treated surgically for vulvodynia, had an acute genital infection or inflammation during the trial period or recovered from such an episode within 14 days, had pelvic pain or sensitivity on bimanual examination, were diagnosed with pelvic organ prolapse of any degree, had any significant medical condition, or had a history of abnormal cervix cytology.

Twenty participants were recruited (Figure 1). Each patient was examined by the same vulvar disease specialist who ruled out other causes of dyspareunia and verified the diagnosis of PV by using the Q-tip test and Friedrich's criteria ${ }^{10}$ (extreme pain elicited by applying light pressure with a cotton wide swab on seven fixed points at the introitus).

During this test, patients were asked to rank their pain intensity on a scale from 0 to 10 ( 0 , experiencing no pain; 10, experiencing maximum pain) to document their baseline pain level. ${ }^{12}$

Subsequently, all patients underwent trial manipulation, which involved applying pressure with a device sufficiently wide to stretch the posterior fornix without overstretching it, thereby temporarily providing support to the USL. To identify the posterior fornix, a lubricated narrow speculum was used, and a swab sufficiently wide to support the USLs was inserted through it (Figure S2). After placing the wide swab stick in the posterior fornix, the speculum was immediately removed, leaving the wide swab in its place (Figure S3). Then, we crossed over to perform sham manipulation (insertion of the device to the posterior fornix without applying pressure).

During each manipulation, and while the wide swab stick and supporting device were in the posterior fornix, the Q-tip test was re-performed (Figure 2) and patients were again asked to rank their vestibular foci pain intensity.

To determine if the results were affected by the manipulation order, subjects were computer-randomized into two groups before manipulation. Subjects in the first group (trial first group) underwent trial manipulation first, followed by sham manipulation; however, the second group (sham first group) underwent sham manipulation first, followed by trial manipulation.

After collecting all data, the average pain intensity levels during the different scenarios were calculated for 
each group. We used a Wilcoxon rank-sum test to determine whether the manipulation order affected the differences in pain intensity compared with the baseline pain level. The average pain levels under the different conditions using a paired sample t-test were determined for the following pairs: baseline pain level and trial manipulation pain level (baseline-trial); baseline pain level and sham manipulation pain level (baseline-sham); and trial manipulation pain level and sham manipulation pain level (trial-sham).

Sample size calculation and statistical methods

Because of multiple comparisons, we applied the Bonferroni correction. Statistical significance was set at $1.67 \%(\alpha=0.0167)$. We expected statistically significant differences resulting from the baseline-trial and trial-control comparisons and no difference resulting from the baseline-control comparison. A difference between the two conditions was viewed as clinically significant if it reflected a change of at least $30 \%$ in the pain level. Fifteen patients were required for this crossover study. All calculations were performed using IBM SPSS Statistics, version 25.

\section{Results}

Women were enrolled at the colposcopy clinic between August 1, 2017 and January 30, 2018. Of the 20 patients who agreed to participate in the study, three did not fulfil the inclusion criteria. There were no differences in the background variables (Table 1) or baseline pain levels $(6.7 \pm 2.2$ units versus $6.4 \pm 3.0$ units, respectively; two-tailed Wilcoxon rank test $\mathrm{p}=0.686$; data not tabulated) of the sham first group and trial first group. We used Cronbach's alpha test to confirm the statistical validity of calculating the average pain intensity from the various foci under each condition (Table S1). Then, we tested whether the manipulation order affected the difference between the baseline pain level and trial manipulation pain level. For the seven women in the trial first group, the average change in pain intensity was 0.86 . However, for the 10 women in the sham first group, the average change was $1.5(\mathrm{P}<.512)$. Therefore, the manipulation order did not have a significant impact on the change in pain levels. Following these results, data from all subjects were gathered in one group. Then, we compared the average pain levels (Table 2) and showed that posterior fornix pressure led to a statistically significant decrease in the average pain level elicited by the vestibular Q-tip test compared with the sham manipulation pain level and baseline pain level (decreases of 1.12 and 1.13 units in pain intensity, respectively; $\mathrm{P}=.003$ ). There was no significant difference between the average baseline pain level at baseline and sham manipulation pain level $(\mathrm{P}=.332)$, as anticipated. However, the overall decrease in the average pain level during trial manipulation was $18.4 \% \pm 2.2 \%$, which was below the $30 \%$ threshold that we considered clinically significant (Table S2). Further examinations of the data revealed that the decrease in the average pain level ranged between $0 \%$ and $78 \%$; in other words, there was at least one subject who experienced no influence of trial manipulation and some subjects who experienced a significant influence. Then, we calculated the overall decrease in the average pain level for the 11 patients who reported any degree of improvement during trial manipulation, resulting in an average change of $28.4 \% \pm 2.2 \%$. Five patients experienced more than $30 \%$ improvement in their pain level.

\section{Discussion}

\section{Main Findings}

By mechanically supporting the USL, we were able to temporarily alleviate provoked vulvar pain in some women. These results support our hypothesis and possibly create a new field in the study of PV.

During trial manipulation for USL support, patients reported a significant reduction in pain intensity compared with their baseline pain level. The wide swab probably sufficiently supported USL laxity to restore its ability to mechanically support the nerve plexuses, thereby relieving the pain (Figure S1).

Although the improvement with USL mechanical support was impressive, several patients described no improvement whatsoever in their pain level. These findings are in accordance with the current consensus, suggesting that vulvodynia is associated with different contributing factors. ${ }^{1}$

In line with this consensus, it is reasonable to suggest that the subjects who reported pain alleviation do, in 
fact, have USL laxity-associated PV.

Our hypothesis was based not only on our own experimental studies ${ }^{8,9}$ but also on a general hypothesis of CPPU caused by weakened USLs ${ }^{6}$ with CPPU found to be curable either by USL plication ${ }^{11}$ or by a posterior sling during a randomized trial involving 1420 women. ${ }^{13}$ The same concept of the role of the USLs was first published in the German literature in 1938 by one of the most famous German gynecologists of the $20^{\text {th }}$ century, Heinrich Martius. ${ }^{14}$ Martius wrote that in $30 \%$ of cases, backaches and pelvic pain were provoked by damage of the paired "ligamenta sacro-uterina" (USL) and the Frankenhauser and sacral ganglia were involved in causing chronic pelvic pain because of the inability of weakened USLs to support them. ${ }^{14}$

The mechanism by which strengthening the USLs in women without overt pelvic organ prolapse alleviates vestibular sensitivity is consistent with that previously proposed (Figure S1). ${ }^{5,6,8,9,14}$ Lax USLs cannot mechanically support the nerve plexuses. Furthermore, vulvodynia may be only one phenotype of T10-L2 and S2-4 referred nerve pain. Nevertheless, it remains unknown why some patients did not experience pain relief from USL mechanical support by the swab. A method that provides broader support to include both USLs (such as the lower blade of a bivalve speculum) may, in fact, alleviate pain in more women. ${ }^{14}$ This was not an option during our study; however, because the handle of the speculum covered the vestibule and prevented Q-tip testing, the device had to be sufficiently narrow to be inserted through the allodynic vulvar vestibule.

Our method of USL support may further support an already established associated factor of PV, namely, musculoskeletal dysfunction such as pelvic muscle overactivity, myofascial changes, and biomechanical changes. ${ }^{1,4}$ It has been repeatedly shown that physical therapy is helpful for alleviating some cases of PV. ${ }^{4}$ Musculoskeletal dysfunction of the pelvic muscles can be caused by USL laxity. It has been demonstrated by video, X-rays, myograms, and electromyography ${ }^{15,16}$ that three oppositely acting directional forces, forward, backward, and downward, act against pubourethral ligaments anteriorly and USLs posteriorly to control bladder continence and evacuation. These forces are equally balanced in the region of the bladder neck. ${ }^{17}$ If USLs are loose, then the posterior forces weaken and the system becomes unbalanced; the forward force (the anterior portion of pubococcygeus muscles) overcompensates by contracting harder to the extent that it can narrow the urethra. ${ }^{17}$ These are the "overactive" muscles addressed by physical therapy. ${ }^{4}$ These findings ${ }^{17,18}$ adequately explain the link between our hypothesis and pelvic muscle dysfunction. ${ }^{1,4}$

\section{Limitations and strengths}

This study was not without limitations. The low number of patients who agreed to participate in this study impacted the statistical power of the findings. Limiting participation to ages younger than 35 years resulted in our findings being inapplicable to older patients and patients with overt pelvic organ prolapse. Nevertheless, this was also a strength of this study because we intended to determine whether USL laxity could be a risk factor associated with vulvodynia in young women without overt pelvic organ prolapse.

\section{Interpretations}

We propose that our procedure of mechanically supporting the USLs with a sufficiently wide device may be used as a readily available clinical test of the existence of USL laxity, not only for patients with vulvodynia but also for those with chronic pelvic pain of unknown origin as well as urgency. ${ }^{14}$ However, this association needs to be examined further.

\section{Conclusions}

Our findings of this randomized, controlled, pilot trial support the hypothesis that USL laxity might be a cause of pelvic floor dysfunction leading to PV. These results may encourage further studies because of their potential therapeutic implications.

Disclosure of Interests : The authors declare no conflict of interest.

\section{Contribution to Authorship:}


MS had a role in Substantial contributions to conception and design, and acquisition of data, and analysis and interpretation of data; AND Drafting the article and revising it critically for important intellectual content; AND Final approval of the version to be published; AND Agreement to be accountable for all aspects of the work in ensuring that questions related to the accuracy or integrity of any part of the work are appropriately investigated and resolved.

PP had a role in Substantial contributions to conception and design, and analysis and interpretation of data; AND Drafting the article and revising it critically for important intellectual content; AND Final approval of the version to be published; AND Agreement to be accountable for all aspects of the work in ensuring that questions related to the accuracy or integrity of any part of the work are appropriately investigated and resolved..

JB had a role in Substantial contributions to conception and design, and acquisition of data, and analysis and interpretation of data; AND Drafting the article and revising it critically for important intellectual content; AND Final approval of the version to be published; AND Agreement to be accountable for all aspects of the work in ensuring that questions related to the accuracy or integrity of any part of the work are appropriately investigated and resolved..

All authors accept responsibility for the paper as published.

IRB approval was received from the Helsinki Committee of the Galilee Medical Center of the Israeli Health Ministry, on July 23, 2017 (authorization number: 0043-17-NHR). ClinicalTrials.gov Identifier: NCT03197337.

\section{Funding: None \\ REFERENCES}

1. Bornstein J, Goldstein AT, Stockdale C, Bergeron S, Pukall C, Zolnoun D, et al. Consensus terminology and classification of persistent vulvar pain and vulvodynia. Obstet Gynecol 2016;127:745-51.

2. Arnold LD, Bachmann GA, Rosen R, Rhoads GG. Assessment of vulvodynia symptoms in a sample of US women: a prevalence survey with a nested case control study. Am J Obstet Gynecol 2007;196:e1-6.

3. Bornstein J, Goldschmid N, Sabo E. Hyperinnervation and mast cell activation may be used as histopathologic diagnostic criteria for vulvar vestibulitis. Gynecol Obstet Invest 2004;58:171-8.

4. Morin M, Carroll MS, Bergeron S. Systematic review of the effectiveness of physical therapy modalities in women with provoked vestibulodynia. Sex Med Rev 2017;5:295-322.

5. Petros PE. Severe chronic pelvic pain in women may be caused by ligamentous laxity in the posterior fornix of the vagina. Aust NZ J Obstet Gynaecol 1996;36:351-4.

6. Marcus Braun N, von Theobold P. Chronic pelvic pain caused by laxity of the uterosacral ligaments: are the posterior fornix syndrome and the Allen-Masters syndrome synonyms? Pain Medicine 2016;17:370371.

7. Wesselmann U, Burnett AL, Heinberg LJ. The urogenital and rectal pain syndromes. Pain. 1997;73:26994

8. Petros P, Bornstein J. Re: vulvar vestibulitis may be a referred pain arising from laxity in the uterosacral ligaments: a hypothesis based on three prospective case reports. Aust N Z J Obstet Gynaecol 2004;44:484-5.

9. Bornstein J, Zarfati D, Petros P. Causation of vulvar vestibulitis. Aust N Z J Obstet Gynaecol 2005; $45: 538-9$.

10. Friedrich EG Jr. Vulvar vestibulitis syndrome. J Reprod Med 1987;32:110-4.

11. Marinoff SC, Turner ML. Vulvar vestibulitis syndrome: an overview. Am J Obstet Gynecol 1991;165:1228-33.

12. Farrar JT, Young JP, Lamoreaux L, Werth JL, Poole MR. Clinical importance of changes in chronic pain intensity measured on an 11-point numerical pain rating scale. Pain 2001;94:149-58.

13. Wagenlehner F, Muller-Funogea I-A, Perletti G, et al. Vaginal apical prolapse repair using two different sling techniques improves chronic pelvic pain, urgency and nocturia- a multicentre study of 1420 
patients. Pelviperineology 2016;35:99-104.

14. Martius H. Über einen häufigen gynäkologischen, Symptomkomplex. Arch Gynecol Obstet 1938;166:332-5.

15. Wu QI, Luo LA, Petros PE. Case report: Mechanical support of the posterior fornix relieved urgency and suburethral tenderness. Pelviperineology $2013 ; 32: 55-6$

16. Petros PE, Ulmsten U. Role of the pelvic floor in bladder neck opening and closure: I muscle forces. Int J Urogynecol Pelvic Floor 1997;8:74-80.

17. Petros PE, Ulmsten U. Role of the pelvic floor in bladder neck opening and closure: II vagina. Int J Urogynecol Pelvic Floor 1997;8:69-73.

18. Petros PE, Abendstein B, Swash M. Retention of urine in women is alleviated by uterosacral ligament repair: implications for Fowler's syndrome. Cent Eur J Urol. 2018;4:436-43.

Figure legends:

Figure 1 : Study flow diagram.

Figure 2: With the wide swab stick in the posterior fornix, the Q-tip test for diagnosing provoked vulvodynia was performed by applying light pressure with a wet Q-tip applicator on seven vestibular foci corresponding with $1,3,5,6,7,9$, and 11 o'clock. The subjective elicited pain was recorded using a numeric rating scale of 0 to 10 .

Figure S1: The ganglia of the Frankenhauser and sacral plexuses are supported by uterosacral ligaments (USLs) at their uterine end. The nerves may be stimulated by gravity or by pelvic organ prolapse to fire, which can be perceived as pain by the cortex. Supporting ligament laxity with a wide swab tensions USLs sufficiently to support the nerve plexuses, thereby relieving vulvodynia pain. L, ligament laxity.

Figure S2 : A lubricated narrow speculum was used to identify the posterior fornix, and a wide swab stick was inserted through it.

Figure S3 : The speculum was removed, and the wide swab stick was left in the posterior fornix. During trial manipulation, the examiner applied pressure with a swab sufficiently wide to support the posterior fornix, thereby temporarily providing support to the uterosacral ligaments. During sham manipulation, the examiner inserted the wide swab stick to the posterior fornix without touching it.

Table titles:

Table 1. Background variables

Table 2. Differences in pain levels of different conditions across all subjects

Table S1. Average pain level experienced at different foci $(n=17)$

Table S2. Overall decrease in pain level with trial manipulation

\begin{tabular}{llll}
\hline $\mathrm{P}$ (two-sided) & Sham first $\mathrm{n}=10$ & Trial first $\mathrm{n}=7$ & \\
\hline $0.583^{*}$ & $26.2 \pm 5.725(20-35)$ & $24.14 \pm 4.025(18-29)$ & $\begin{array}{l}\text { Age (years) Average } \pm \mathrm{SD} \\
\text { Median (range) } \\
\text { Age at first menstrual } \\
\text { period (years) } \\
\text { Average } \pm \text { SD Median } \\
\text { (range) }\end{array}$ \\
& $12 \pm 1.612(9-14)$ & $13.3 \pm 0.813(12-14)$ & $\begin{array}{l}\text { Use of oral contraception } \\
\text { N (\%) }\end{array}$ \\
$0.622^{* *}$ & $5(50 \%)$ & $5(71.4 \%)$ & $\begin{array}{l}\text { Smoking N }(\%) \\
\text { PV, N (\%) Primary } \\
1.0^{* *}\end{array}$ \\
$0.162^{* *}$ & $1(10 \%)$ & $1(14.3 \%)$ & Secondary
\end{tabular}




\begin{tabular}{llll}
\hline $\mathrm{P}$ (two-sided) & Sham first $\mathrm{n}=10$ & Trial first $\mathrm{n}=7$ & \\
\hline $0.434^{* *}$ & $5(50 \%) 5(50 \%)$ & $2(29 \%) 5(71 \%)$ & $\begin{array}{l}\text { Symptom severity N }(\%) \\
\text { Moderate Severe }\end{array}$ \\
\hline
\end{tabular}

Table 1. Background variables

*Wilcoxon rank sum test. ${ }^{* *}$ Fisher's exact test.

PV, provoked vulvodynia; SD, standard deviation.

$\mathrm{n}=17 \quad \mathrm{P}(\text { two-sided })^{*}$

Difference between trial manipulation and basal pain level (units), average \pm SD $\quad \begin{array}{lll}1.13 \pm 1.34 & 0.003\end{array}$

$\begin{array}{lll}\text { Difference between trial manipulation and sham manipulation, average } \pm \mathrm{SD} & 1.12 \pm 1.34 & 0.003\end{array}$

$\begin{array}{lll}\text { Difference between sham manipulation and basal pain level, average } \pm \text { SD } & 0.01 \pm 0.04 & 0.332\end{array}$

Table 2. Differences in pain levels of different conditions across all subjects

*Paired samples t test.

$\mathrm{SD}$, standard deviation.

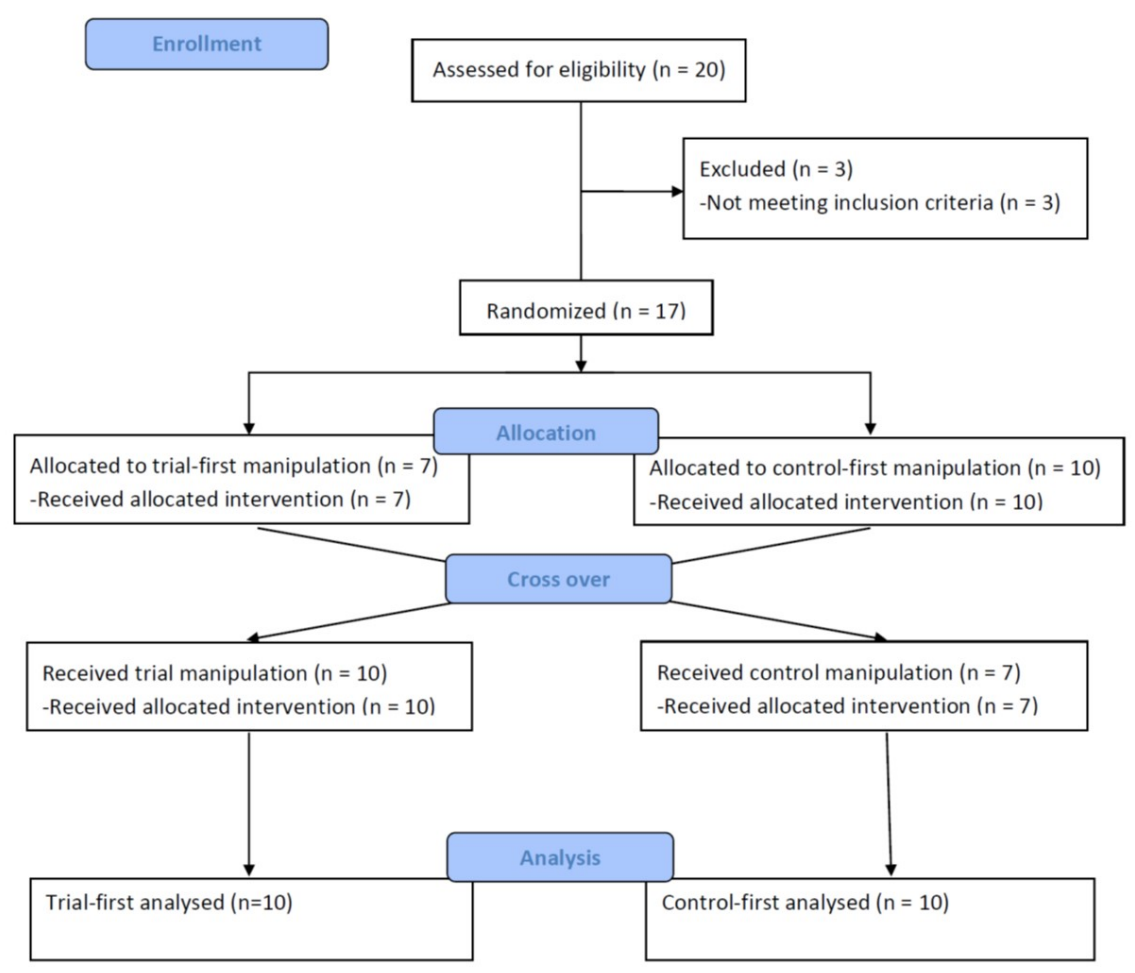




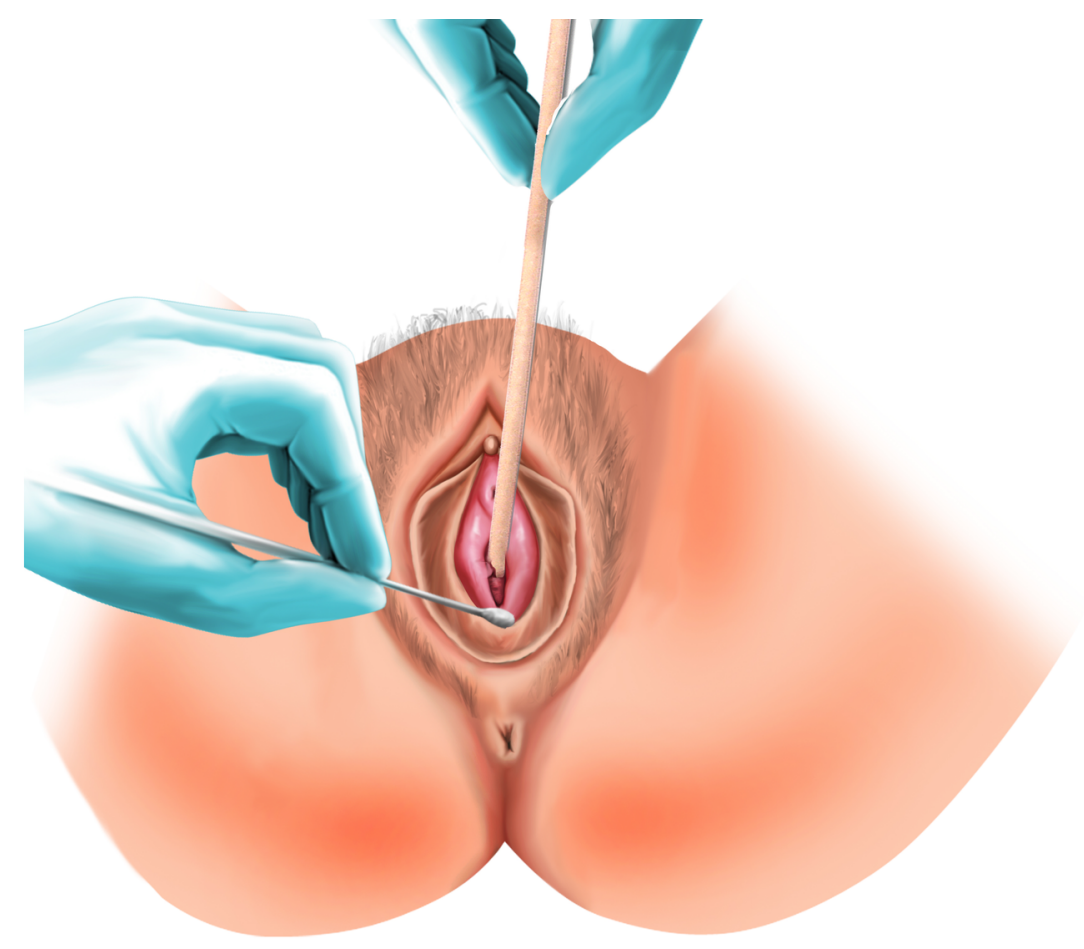

